\title{
Occurrence of Tension Pneumothorax following Fogarty Embo- lectomy Catheter use for Lung Isolation in a Neonate with Con- genital Pulmonary Airway Malformation
}

\section{Budzinski Jessie and Dinesh K Choudhry*}

Department of Anesthesiology, A.I. Dupont Hospital for Children, USA

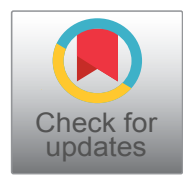

*Corresponding author: Dinesh K Choudhry, M.D., Associate Professor, Department of Anesthesiology, A.I. Dupont Hospital for Children, Wilmington, DE, USA

\begin{abstract}
A pneumothorax can arise in a variety of clinical settings in the operating room, from bleb rupture to iatrogenic injury. Should it expand in size and cause increase in intrapleural pressures with resultant diminished venous return, it becomes referred to as a tension pneumothorax. We report a case of a premature infant presenting for repair of congenital pulmonary airway malformation who suffered an iatrogenic tension pneumothorax during lung isolation with fogarty embolectomy catheter used for single lung ventilation in a neonate.
\end{abstract}

\section{Introduction}

Congenital pulmonary airway malformation (CPAM), formerly known as congenital cystic adenomatoid malformation (CCAM), is one of the most frequent pulmonary malformations that presents as a multicystic mass of pulmonary tissue with proliferation of bronchiolar structures that fail to mature [1]. While the exact pathogenesis is unclear, it has been hypothesized that the cysts develop either because of failure of the bronchial buds to join alveolar mesenchyme [2,3] or from overgrowth of terminal bronchioles. Children presenting with CPAM bring challenges with regard to their anesthetic management, including oxygenation and need for lung isolation. We discuss a neonate presenting with a right-sided CPAM lesion, who developed a tension pneumothorax of the contralateral side, secondary to attempted lung isolation with a fogarty embolectomy catheter.

\section{Case Description}

A 5-day-old, 2.6-kg male born at 36 weeks' gestation

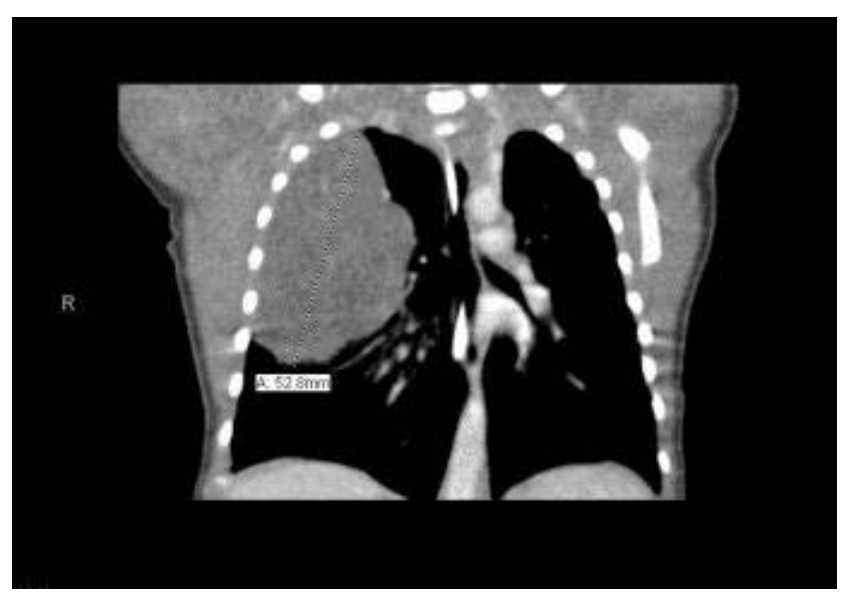

Figure 1: CT of the chest with contrast showing congenital pulmonary airway malformation in the right lung.

via c-section was noticed to have respiratory difficulty shortly after birth. Radiologic investigations revealed a right-sided chest mass with slight mediastinal shift (Figure 1). A diagnosis of CPAM was made, and the child was scheduled for right upper lobectomy. He was otherwise healthy with no abnormal laboratory or clinical findings. The case was discussed with the surgical team, and it was decided to proceed with lung isolation and single-lung ventilation (SLV). Atropine was administered intravenously, and anesthesia was induced with propofol and rocuronium. Mask ventilation was instituted using minimal pressures with $2 \%$ sevoflurane and $100 \%$ oxygen. Direct laryngoscopy (DL) was performed and a $4 \mathrm{Fr}$ Fogarty embolectomy catheter (FC) easily inserted until resistance was met. An initial attempt with

Citation: Jessie B, Dinesh KC (2018) Occurrence of Tension Pneumothorax following Fogarty Embolectomy Catheter use for Lung Isolation in a Neonate with Congenital Pulmonary Airway Malformation. Int J Anesthetic Anesthesiol 5:079. doi.org/10.23937/2377-4630/1410079

Accepted: November 03, 2018: Published: November 05, 2018

Copyright: (C) 2018 Jessie B, et al. This is an open-access article distributed under the terms of the Creative Commons Attribution License, which permits unrestricted use, distribution, and reproduction in any medium, provided the original author and source are credited. 


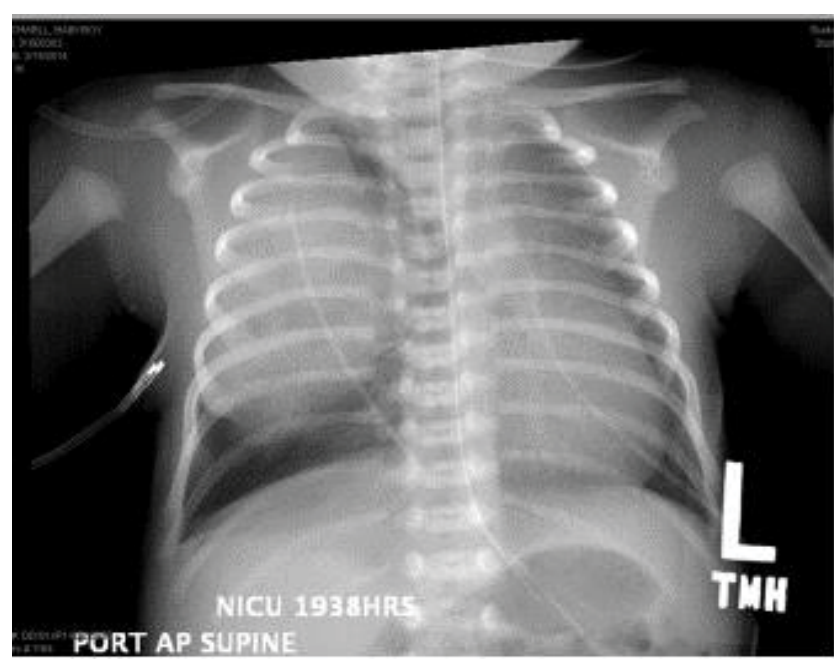

Figure 2: Pneumothorax of the contralateral lung (left) with chest tube in situ.

a 3.0 cuffed tube was met with resistance, and, subsequently, a 3.0 uncuffed endotracheal tube (ETT) was placed alongside the FC. A fiberoptic scope (FOS) was then advanced through the ETT, and we discovered that the FC had entered the unintended left main bronchus. We therefore withdrew the catheter and repositioned it into the operative (right) bronchus. The patient's oxygenation and ventilation were optimal at this point and blood pressure and heart rate within normal ranges. Several minutes thereafter, the end-tidal $\mathrm{CO}_{2}$ tracing diminished and then disappeared. Oxygen saturations then began to fall rather dramatically, and the child developed bradycardia. At this time, ETT position was confirmed and auscultation revealed minimal coarse sounds on the right and absent sounds on the left chest. Acute bronchospasm was suspected, and albuterol and epinephrine were administered. With no resultant improvement, pneumothorax was suspected. A stat portable radiograph was ordered; however, given the rapidly deteriorating condition of the child, it was decided not to wait for the radiograph but place the chest tube entirely on clinical grounds (Figure 2). Immediately upon decompression, we were able to reestablish ventilation and $\mathrm{ETCO}_{2}$ tracing reappeared and oxygenation improved. Hemodynamic parameters returned to physiologic range. In view of the life-threatening episode that had occurred and nonurgent nature of the procedure, a decision was made to postpone surgery. The child was transported back to the neonatal intensive care unit intubated and ventilated in stable condition.

\section{Discussion}

Congenital pulmonary airway malformation usually presents in one of three ways: Respiratory distress of the newborn, recurrent pneumonia in young children, or incidentally in asymptomatic adults [4]. Should a newborn present with CPAM, initiation of breathing after delivery may lead to gas trapping and further cystic expansion with worsening respiratory compromise. Most CPAM lesions require resection of the mass either thoraco- scopically or by open thoracotomy, and prognosis after surgical excision is generally good. With increasing popularity of thorcoscopic approach to lung surgery, SLV is requested for an increasing spectrum of surgical procedures in infants and children. Advances in anesthetic equipment, lung blocking devices, monitoring, technology, and pharmacology have increased the safety of one-lung anesthesia. Despite these advances, injuries are associated with SLV devices, such as double-lumen tubes (DLT) and endobronchial blockers. To date, there is only one other documented case of accidental bronchial injury with FC during lung isolation likely related to overdistension of the FC balloon, which was noticed by the surgical team during thoracotomy. In this instance, there were no hemodynamic sequelae, and the lesion was repaired intraoperatively [5]. Literature comparing injuries in adult patients who have been intubated with DLT show more frequent complications such as hoarseness and vocal cord lesions compared with those who had single-lumen tubes with blocking devices [6]. To our knowledge, this is the first case where injury to the contralateral healthy lung has occurred because of FC used for lung isolation.

Infants and neonates, because of their size, are unable to accommodate either a DLT or Arndt blocker. Lung isolation in this age group is accomplished either by endobronchial advancement of the ETT to the ventilated nonsurgical lung or by placing an FC alongside the ETT to block the surgical lung [7]. The latter technique offers the advantage of accurate lung block and obviates the need for ETT withdrawal into the trachea when expansion of the collapsed lung needs to be reestablished. The FC has been used at our hospital for SLV in infants for many years and has worked quite well without any complications. It is advanced into the trachea by DL, and the ETT is then placed alongside the blocker. The blocker is then accurately positioned in the desired main stem bronchus with the help of FOS advanced through the ETT, and the blocker balloon is inflated with air under fiberoptic view [8].

The tension pneumothorax in this scenario is the likely result of mechanical injury from the FC that was used for lung isolation. Another possible cause however is barotrauma secondary to positive pressure ventilation that was commenced after the child was intubated. We feel that trauma due to the FC to be more likely because pneumothorax occurred soon after the blocker was withdrawn from the left lung and positioned in the right lung. The FC has a considerably stiff metal stylet, and it is quite likely that, as we were trying to advance the ETT alongside the blocker, we may have inadvertently pushed the blocker deeper into the lung that led to perforation of one of the smaller more distal bronchi. This was not clinically apparent as long as the blocker was sealing the hole, but, when the blocker was withdrawn from the left and redirected to the correct side, the puncture site was no longer occluded and pneumo- 
thorax resulted. We feel that the occurrence of pneumothorax was likely due to injury to the healthy lung caused by stiff stylet in the FC and not due to the underlying CPAM. Also, the literature does not support that a child with CPAM is more prone to pneumothorax on the contralateral unaffected side; instead, it is more likely on the ipsilateral side [9]. However, with initiation of mechanical ventilation in a patient with a large cystic lesion on the right side, it is possible most of the pressure was directed to the unaffected (left) side resulting in its overinflation and consequent tension pneumothorax.

We feel that this untoward event brings some insight into modifications that may be advisable with the FC use while managing these children. First, when the blocker is being placed in the trachea, it should not be advanced too far in until the resistance is met, as has been our practice for many years. It should be advanced only 2 to $3 \mathrm{~cm}$ beyond the tip of the ETT into the trachea so that it can be visualized with the FOS and positioned in the desired bronchus under vision. Second, as the ETT is advanced alongside the blocker, special care is required to ensure that the blocker is held stationary and does not inadvertently get pushed deeper into the lung along with the ETT and cause injury. We have on previous occasions considered the possibility of removing the stylet from the FC before advancing it into the trachea, but we find that the blocker is so soft and floppy without the stylet that it is impossible to manipulate it to change its position when the ETT has been placed alongside it. Last but not the least, it may be advisable for the surgery team to be in the operating room and have a chest tube readily available so that if pneumothorax does occur, no time is wasted in chest tube insertion that could be life saving in such a situation.

In conclusion, the FC works well as a device for lung isolation in infants and children. However, potential for injury still exists, and special care and precautions are needed to ensure its safe use until a better alternative is available to accomplish lung isolation in this age group [10].

\section{Funding}

This work did not receive funding from the NIH.

\section{References}

1. Lezmi G, Hadchouel A, Khen-Dunlop N, Vibhushan S, Benachi A, et al. (2013) Congenital cystic adenomatoid malformations of the lung: Diagnosis, treatment, pathophysiological hypothesis. Rev Pneumol Clin 69: 190-197.

2. Prendergrast B, Fernando AM, Mankad PS (1998) Congenital cystic adenoid malformation in a pre-term infant: Management considerations. Pediatr Surg Int 14: 92-93.

3. Correia-Pinto J, Gonzaga S, Huang Y, Rottier R (2010) Congenital lung lesions--underlying molecular mechanisms. Semin Pediatr Surg 19: 171-179.

4. Laberge JM, Flageole $H$, Pugash D, Khalife S, Blair G, et al. (2001) Outcome of the prenatally diagnosed congenital cystic adenomatoid lung malformation: A Canadian experience. Fetal Diagn Ther 16: 178-186.

5. Borchardt RA, LaQuaglia MP, McDowall RH, Wilson RS (1998) Bronchial injury during lung isolation in a pediatric patient. Anesth Analg 87: 324-325.

6. Knoll H, Ziegeler S, Schreiber JU, Buchinger $\mathrm{H}$, Bialas $\mathrm{P}$, et al. (2006) Airway injuries after one-lung ventilation: A comparison between double-lumen tube and endobronchial blocker: A randomized, prospective, controlled trial. Anesthesiology 105: 471-477.

7. Choudhry DK (2005) Single-lung ventilation in pediatric anesthesia. Anesthesiol Clin North America 23: 693-708.

8. Hammer GB (2002) Pediatric thoracic anesthesia. Anesthesiol Clin North America 20: 153-180.

9. Miller JA, Corteville JE, Langer JC (1996) Congenital cystic adenomatoid malformation in the fetus: Natural history and predictors of outcome. J Pediatr Surg 31: 805-808.

10. Dolgin SE (2012) Surgical care of major newborn malformations. Hackensack, NJ: World Scientific Publishing Co, 239-260. 